\title{
FUNDAMENTAL SOLUTION OF COMPOSITE THICK SHELLS WITH CLAMPED EDGES
}

\author{
JIAO JIN ${ }^{1}$, HUI WEI ${ }^{1}$, JIANLONG ZHENG $^{1}$ \& PIHUA WEN $^{2}$ \\ ${ }^{1}$ School of Traffic and Transportation Engineering, Changsha University of Science and Technology, China \\ ${ }^{2}$ School of Engineering and Materials Science, Queen Mary University of London, UK
}

\begin{abstract}
In this paper, a rectangular composites double curved shell with four clamped edges is studied under static distributed and concentrated loads. The governing equations for the laminated and functionally graded shells with respect to the middle surface are presented, and the fundamental solutions are obtained. The exact solutions of the laminated and functionally graded shells should serve as the benchmark solutions for any numerical computation methods and can be used in the boundary element method and meshless method.
\end{abstract}

Keywords: fundamental solution, composite and functionally graded materials, plate and shell, moderate thick plate theory.

\section{INTRODUCTION}

A number of theories exist for layered anisotropic shells [1] and many of them were developed for thin shells based on the Kirchhoff hypothesis. However, the application of thin plate theory could lead to $30 \%$ or more errors to layered anisotropic composite shells [2]. The higher-order theory of Reddy and Liu [3] for composite shells is based on five degrees of freedom. This theory assumes a constant transverse deflection through the thickness and the displacements of the middle surface are expanded, as cubic functions of the thickness coordinate. Exact solutions were derived for simply supported laminated shells under static loads by Reddy [2], using double Fourier transform technique. This paper deals with the analytical solutions of the laminated and orthotropic functionally graded, cross-ply laminated, double curved shells with four clamped edges under static transverse concentrated loads. Analytical solutions for transverse deflection, in-plane tensile force and moment are presented for the laminated and various functionally graded shells. The exact solutions presented herein for cylindrical and spherical functionally graded shells under uniformly distributed load and concentrated force can be used as the benchmark results for any numerical methods, such as the finite element, finite difference, boundary element and meshless methods.

\section{GOVERNING EQUATIONS FOR ORTHOTROPIC SHELLS}

An uniform thickness shell is shown in Fig. 1, where $\xi_{1}, \xi_{2}$ and $\varsigma$ denote the orthogonal curvilinear coordinates, where $\xi_{1}$ and $\xi_{2}$ are the lines of curvature on the middle surface $\varsigma=0$. For cylindrical and spherical shells, the lines of principal curvature coincide with the coordinate axis. With the assumptions of a moderate thick shell theory, one has the following displacement fields in the time domain

$$
\begin{gathered}
u_{1}\left(\xi_{1}, \xi_{2}, \varsigma\right)=u_{0}\left(\xi_{1}, \xi_{2}\right)+\varsigma \varphi_{1}\left(\xi_{1}, \xi_{2}\right), \\
u_{2}\left(\xi_{1}, \xi_{2}, \varsigma\right)=v_{0}\left(\xi_{1}, \xi_{2}\right)+\varsigma \varphi_{2}\left(\xi_{1}, \xi_{2}\right), \\
u_{3}\left(\xi_{1}, \xi_{2}, \varsigma\right)=w_{0}\left(\xi_{1}, \xi_{2}\right),
\end{gathered}
$$




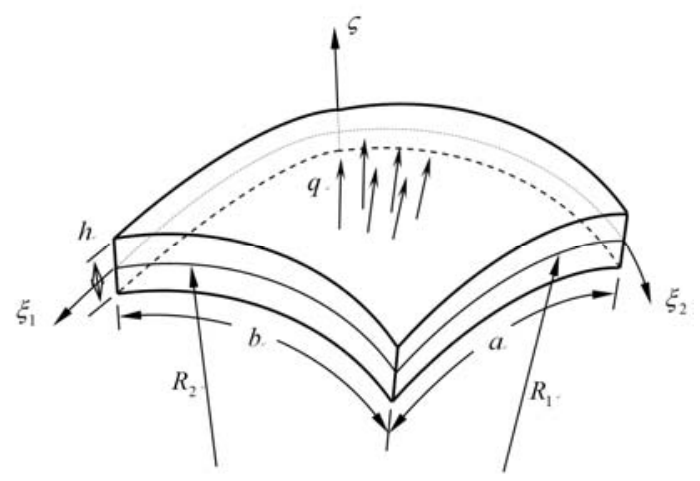

Figure 1: Geometry of a double curved shell.

where $\left(u_{0}, v_{0}, w_{0}\right)$ are the displacements of the middle surface and $\left(\varphi_{1}, \varphi_{2}\right)$ the rotations of a normal to the $\xi_{1}$ and $\xi_{2}$ axes respectively. In the following analysis coordinate axis is $(x, y)$ for convenience. The resultants per unit length can be derived by

$$
\begin{gathered}
\left(N_{i}, M_{i}\right)=\int_{-h / 2}^{h / 2} \sigma_{i}(x, y, z)(1, z) d z, i=1,2,6 ; \\
Q_{1}=\kappa \int_{-h / 2}^{h / 2} \sigma_{4}(x, y, z) d z, Q_{2}=\kappa \int_{-h / 2}^{h / 2} \sigma_{5}(x, y, z) d z,
\end{gathered}
$$

where $\sigma_{1}=\sigma_{x}, \sigma_{2}=\sigma_{y}, \sigma_{6}=\sigma_{x y}, \sigma_{4}=\sigma_{y z}, \sigma_{5}=\sigma_{x z}$ and $\kappa$ denotes the shear correction factor ( $5 / 6$ for the Reissner's moderate thick plate theory). The stress-strain relations are given, in the shell coordinate system, as

$$
\left\{\begin{array}{l}
\sigma_{1} \\
\sigma_{2} \\
\sigma_{4} \\
\sigma_{5} \\
\sigma_{6}
\end{array}\right\}=\left[\begin{array}{ccccc}
Q_{11} & Q_{12} & 0 & 0 & 0 \\
Q_{12} & Q_{22} & 0 & 0 & 0 \\
0 & 0 & Q_{44} & 0 & 0 \\
0 & 0 & 0 & Q_{55} & 0 \\
0 & 0 & 0 & 0 & Q_{66}
\end{array}\right]\left\{\begin{array}{c}
\varepsilon_{1} \\
\varepsilon_{2} \\
\varepsilon_{4} \\
\varepsilon_{5} \\
\varepsilon_{6}
\end{array}\right\},
$$

where $Q_{i j}$ are the stiffness of orthotropic shell which can be determined by the engineering constants as

$$
Q_{11}=\frac{E_{1}}{1-v_{12} v_{21}}, Q_{12}=\frac{v_{12} E_{2}}{1-v_{12} v_{21}}, Q_{22}=\frac{E_{2}}{1-v_{12} v_{21}}, Q_{66}=G_{12}, Q_{44}=G_{23}, Q_{55}=G_{13},
$$

in which $E, v$ and $G$ are Young's moduli, Poisson's ratio and shear moduli respectively. Substituting (3) and (4) into (2) gives the stress resultants, ( $N_{6}=N_{12}, M_{6}=M_{12}$ for convenience), as

$$
N_{1}=A_{11}\left(\frac{\partial u_{0}}{\partial x}+\frac{w_{0}}{R_{1}}\right)+A_{12}\left(\frac{\partial v_{0}}{\partial y}+\frac{w_{0}}{R_{2}}\right)+B_{11} \frac{\partial \varphi_{1}}{\partial x}+B_{12} \frac{\partial \varphi_{2}}{\partial y}
$$




$$
\begin{gathered}
N_{2}=A_{12}\left(\frac{\partial u_{0}}{\partial x}+\frac{w_{0}}{R_{1}}\right)+A_{22}\left(\frac{\partial v_{0}}{\partial y}+\frac{w_{0}}{R_{2}}\right)+B_{12} \frac{\partial \varphi_{1}}{\partial x}+B_{22} \frac{\partial \varphi_{2}}{\partial y} \\
N_{6}=A_{66}\left(\frac{\partial u_{0}}{\partial y}+\frac{\partial v_{0}}{\partial x}\right)+B_{66}\left[\frac{\partial \varphi_{1}}{\partial y}+\frac{\partial \varphi_{2}}{\partial x}+C_{0}\left(\frac{\partial u_{0}}{\partial y}-\frac{\partial v_{0}}{\partial x}\right)\right], \\
M_{1}=B_{11}\left(\frac{\partial u_{0}}{\partial x}+\frac{w_{0}}{R_{1}}\right)+B_{12}\left(\frac{\partial v_{0}}{\partial y}+\frac{w_{0}}{R_{2}}\right)+D_{11} \frac{\partial \varphi_{1}}{\partial x}+D_{12} \frac{\partial \varphi_{2}}{\partial y} \\
M_{2}=B_{12}\left(\frac{\partial u_{0}}{\partial x}+\frac{w_{0}}{R_{1}}\right)+B_{22}\left(\frac{\partial v_{0}}{\partial y}+\frac{w_{0}}{R_{2}}\right)+D_{12} \frac{\partial \varphi_{1}}{\partial x}+D_{22} \frac{\partial \varphi_{2}}{\partial y} \\
M_{6}=B_{66}\left(\frac{\partial u_{0}}{\partial y}+\frac{\partial v_{0}}{\partial x}\right)+D_{66}\left[\frac{\partial \varphi_{1}}{\partial y}+\frac{\partial \varphi_{2}}{\partial x}+C_{0}\left(\frac{\partial u_{0}}{\partial y}-\frac{\partial v_{0}}{\partial x}\right)\right],
\end{gathered}
$$

where the stiffness coefficients are defined as

$$
A_{i j}=\int_{-h / 2}^{h / 2} Q_{i j} d z, \quad B_{i j}=\int_{-h / 2}^{h / 2} Q_{i j} z d z, \quad D_{i j}=\int_{-h / 2}^{h / 2} Q_{i j} z^{2} d z, \quad i, j=1,2,4,5,6 .
$$

For the laminated shells, we have

$$
A_{i j}=\sum_{k=1}^{n} Q_{i j}^{(k)}\left(z_{k+1}-z_{k}\right), \quad B_{i j}=\frac{1}{2} \sum_{k=1}^{n} Q_{i j}^{(k)}\left(z_{k+1}^{2}-z_{k}^{2}\right), \quad D_{i j}=\frac{1}{3} \sum_{k=1}^{n} Q_{i j}^{(k)}\left(z_{k+1}^{3}-z_{k}^{3}\right),
$$

where $Q_{i j}^{(k)}$ denotes the lamina stiffness referred to the material coordinates of the $k$-th lamina and $n$ is the number of layers in the laminate. For the gradation of material properties along the plate thickness, we assume the variation profile for volume fraction obey [4]

$$
P(z)=P_{b}+\left(P_{t}-P_{b}\right)(z / h+0.5)^{\eta},
$$

where $P$ denotes a generic property like modulus, $P_{t}$ and $P_{b}$ denote the property on the top and the bottom surfaces respectively, and $\eta$ is a parameter that dictates the material variation profile through the thickness. Poisson ratios and density of mass are assumed to be uniform. In this case, all material constants, from (7), become

$$
\begin{gathered}
A_{i j}=\frac{(\eta+\beta)}{2(\eta+1)} Q_{i j}^{(b)} h, \quad B_{i j}=\frac{\eta(\beta-1)}{2(\eta+1)(\eta+2)} Q_{i j}^{(b)} h^{2}, \\
D_{i j}=\frac{\eta\left(\eta^{2}+3 \eta+8\right)+3 \beta\left(\eta^{2}+\eta+2\right)}{12(\eta+1)(\eta+2)(\eta+3)} Q_{i j}^{(b)} h^{3}, \quad i, j=1,2,4,5,6,
\end{gathered}
$$

where $\beta=P_{t} / P_{b}$ and $Q_{i j}^{(b)}$ are material stiffness on the bottom surface. Furthermore, we can also assume that the exponential gradation of material properties in thickness direction, 


$$
P(z)=P_{b} e^{\alpha(z / h+0.5)},
$$

where $\alpha=\ln (\beta)$ and hence $e^{\alpha}=\beta$. Then material constants can be written as

$$
\begin{gathered}
A_{i j}=\frac{\left(-1+e^{\alpha}\right)}{\alpha} Q_{i j}^{(b)} h, B_{i j}=\frac{\alpha+2+(\alpha-2) e^{\alpha}}{2 \alpha^{2}} Q_{i j}^{(b)} h^{2}, \\
D_{i j}=\frac{-\alpha^{2}-4 \alpha-8+\left(\alpha^{2}-4 \alpha+8\right) e^{\alpha}}{4 \alpha^{3}} Q_{i j}^{(b)} h^{3} .
\end{gathered}
$$

Use of the modified strain-displacements relations in Hamilton's principal yields the following equilibrium equations [5]

$$
\begin{gathered}
\frac{\partial N_{1}}{\partial x}+\frac{\partial}{\partial y}\left(N_{6}+C_{0} M_{6}\right)+\frac{Q_{1}}{R_{1}}=0 \\
\frac{\partial}{\partial x}\left(N_{6}-C_{0} M_{6}\right)+\frac{\partial N_{2}}{\partial y}+\frac{Q_{2}}{R_{2}}=0 \\
\frac{\partial Q_{1}}{\partial x}+\frac{\partial Q_{2}}{\partial y}-\left(\frac{N_{1}}{R_{1}}+\frac{N_{2}}{R_{2}}\right)+q=0 \\
\frac{\partial M_{1}}{\partial x}+\frac{\partial M_{6}}{\partial y}-Q_{1}=0 \\
\frac{\partial M_{6}}{\partial x}+\frac{\partial M_{2}}{\partial y}-Q_{2}=0
\end{gathered}
$$

The equations of motion can be expressed in terms of the displacement as

$$
\begin{aligned}
& A_{11}\left(\frac{\partial^{2} u_{0}}{\partial^{2} x}+\frac{1}{R_{1}} \frac{\partial w_{0}}{\partial x}\right)+A_{12}\left(\frac{\partial^{2} v_{0}}{\partial x \partial y}+\frac{1}{R_{2}} \frac{\partial w_{0}}{\partial x}\right)+B_{11} \frac{\partial^{2} \varphi_{1}}{\partial^{2} x}+B_{12} \frac{\partial^{2} \varphi_{2}}{\partial x \partial y}+A_{66}\left(\frac{\partial^{2} v_{0}}{\partial x \partial y}+\frac{\partial^{2} u_{0}}{\partial^{2} y}\right) \\
& \left(B_{66}+C_{0} D_{66}\right)\left[\frac{\partial^{2} \varphi_{1}}{\partial^{2} y}+\frac{\partial^{2} \varphi_{2}}{\partial x \partial y}+C_{0}\left(\frac{\partial^{2} u_{0}}{\partial^{2} y}-\frac{\partial^{2} v_{0}}{\partial x \partial y}\right)\right]+C_{0} B_{66}\left(\frac{\partial^{2} v_{0}}{\partial x \partial y}+\frac{\partial^{2} u_{0}}{\partial^{2} y}\right) \\
& +\frac{\kappa A_{55}}{R_{1}}\left(\frac{\partial w_{0}}{\partial x}+\varphi_{1}-\frac{u_{0}}{R_{1}}\right)=0, \\
& A_{12}\left(\frac{\partial^{2} u_{0}}{\partial x \partial y}+\frac{1}{R_{1}} \frac{\partial w_{0}}{\partial y}\right)+A_{22}\left(\frac{\partial^{2} v_{0}}{\partial^{2} y}+\frac{1}{R_{2}} \frac{\partial w_{0}}{\partial y}\right)+B_{12} \frac{\partial^{2} \varphi_{1}}{\partial x \partial y}+B_{22} \frac{\partial^{2} \varphi_{2}}{\partial^{2} y}+A_{66}\left(\frac{\partial^{2} v_{0}}{\partial^{2} x}+\frac{\partial^{2} u_{0}}{\partial x \partial y}\right) \\
& \left(B_{66}-C_{0} D_{66}\right)\left[\frac{\partial^{2} \varphi_{1}}{\partial x \partial y}+\frac{\partial^{2} \varphi_{2}}{\partial^{2} x}+C_{0}\left(\frac{\partial^{2} u_{0}}{\partial x \partial y}-\frac{\partial^{2} v_{0}}{\partial^{2} x}\right)\right]-C_{0} B_{66}\left(\frac{\partial^{2} v_{0}}{\partial^{2} x}+\frac{\partial^{2} u_{0}}{\partial x \partial y}\right) \\
& +\frac{\kappa A_{44}}{R_{1}}\left(\frac{\partial w_{0}}{\partial y}+\varphi_{2}-\frac{v_{0}}{R_{2}}\right)=0,
\end{aligned}
$$




$$
\begin{gathered}
\kappa A_{55}\left(\frac{\partial^{2} w_{0}}{\partial^{2} x}+\frac{\partial \varphi_{1}}{\partial x}-\frac{1}{R_{1}} \frac{\partial u_{0}}{\partial x}\right)+\kappa A_{44}\left(\frac{\partial^{2} w_{0}}{\partial^{2} y}+\frac{\partial \varphi_{2}}{\partial y}-\frac{1}{R_{2}} \frac{\partial v_{0}}{\partial y}\right) \\
-\frac{1}{R_{1}}\left[A_{11}\left(\frac{\partial u_{0}}{\partial x}+\frac{w_{0}}{R_{1}}\right)+A_{12}\left(\frac{\partial v_{0}}{\partial y}+\frac{w_{0}}{R_{2}}\right)+B_{11} \frac{\partial \varphi_{1}}{\partial x}+B_{12} \frac{\partial \varphi_{2}}{\partial y}\right] \\
-\frac{1}{R_{2}}\left[A_{12}\left(\frac{\partial u_{0}}{\partial x}+\frac{w_{0}}{R_{1}}\right)+A_{22}\left(\frac{\partial v_{0}}{\partial y}+\frac{w_{0}}{R_{2}}\right)+B_{11} \frac{\partial \varphi_{1}}{\partial x}+B_{12} \frac{\partial \varphi_{2}}{\partial y}\right]+q=0, \\
B_{11}\left(\frac{\partial^{2} u_{0}}{\partial^{2} x}+\frac{1}{R_{1}} \frac{\partial w_{0}}{\partial x}\right)+B_{12}\left(\frac{\partial^{2} v_{0}}{\partial x \partial y}+\frac{1}{R_{2}} \frac{\partial w_{0}}{\partial x}\right)+D_{11} \frac{\partial^{2} \varphi_{1}}{\partial^{2} x}+D_{12} \frac{\partial^{2} \varphi_{2}}{\partial x \partial y}+B_{66}\left(\frac{\partial^{2} v_{0}}{\partial x \partial y}+\frac{\partial^{2} u_{0}}{\partial^{2} y}\right) \\
D_{66}\left[\frac{\partial^{2} \varphi_{1}}{\partial^{2} y}+\frac{\partial^{2} \varphi_{2}}{\partial x \partial y}+C_{0}\left(\frac{\partial^{2} u_{0}}{\partial^{2} y}-\frac{\partial^{2} v_{0}}{\partial x \partial y}\right)\right]-\kappa A_{55}\left(\frac{\partial w_{0}}{\partial x}+\varphi_{1}-\frac{u_{0}}{R_{1}}\right)=0, \\
B_{12}\left(\frac{\partial^{2} u_{0}}{\partial x \partial y}+\frac{1}{R_{1}} \frac{\partial w_{0}}{\partial y}\right)+B_{22}\left(\frac{\partial^{2} v_{0}}{\partial^{2} y}+\frac{1}{R_{2}} \frac{\partial w_{0}}{\partial y}\right)+D_{12} \frac{\partial^{2} \varphi_{1}}{\partial x \partial y}+D_{22} \frac{\partial^{2} \varphi_{2}}{\partial^{2} y}+B_{66}\left(\frac{\partial^{2} v_{0}}{\partial^{2} x}+\frac{\partial^{2} u_{0}}{\partial x \partial y}\right) \\
D_{66}\left[\frac{\partial^{2} \varphi_{1}}{\partial x \partial y}+\frac{\partial^{2} \varphi_{2}}{\partial^{2} x}+C_{0}\left(\frac{\partial^{2} u_{0}}{\partial x \partial y}-\frac{\partial^{2} v_{0}}{\partial^{2} x}\right)\right]-\kappa A_{44}\left(\frac{\partial w_{0}}{\partial y}+\varphi_{2}-\frac{v_{0}}{R_{2}}\right)=0 .
\end{gathered}
$$

\section{ANALYTICAL SOLUTION}

3.1 General solution for rectangular shell with two opposite simply supported edges

Consider a rectangular double curved shell of two dimensions $(a \times b)$ with two opposite simply supported edges, $a$ and $b$ are the dimensions of the shell middle surface along the $x$ and $y$ axes. The boundary conditions on these two edges are expressed, called Case (1), as

$$
\begin{aligned}
& u_{0}(x, 0)=N_{2}(x, 0)=w_{0}(x, 0)=\varphi_{1}(x, 0)=M_{2}(x, 0)=0, \\
& u_{0}(x, b)=N_{2}(x, b)=w_{0}(x, b)=\varphi_{1}(x, b)=M_{2}(x, b)=0 .
\end{aligned}
$$

Then the exact form of the spatial variations of displacement and rotation can be written [6] as

$$
\begin{gathered}
u^{(1)}(x, y)=\sum_{n=1}^{\infty} U_{n}^{(1)}(x) \sin \beta_{n} y m= \\
v^{(1)}(x, y)=\sum_{n=1}^{\infty} V_{n}^{(1)}(x) \cos \beta_{n} y
\end{gathered}
$$




$$
\begin{gathered}
w^{(1)}(x, y)=\sum_{n=1}^{\infty} W_{n}^{(1)}(x) \sin \beta_{n} y, \\
\varphi_{1}^{(1)}(x, y)=\sum_{n=1}^{\infty} X_{n}^{(1)}(x) \sin \beta_{n} y, \\
\varphi_{2}^{(1)}(x, y)=\sum_{n=1}^{\infty} Y_{n}^{(1)}(x) \cos \beta_{n} y,
\end{gathered}
$$

where $\beta_{n}=n \pi / b$. Substituting (15) into (13) gives a set of differential equations with respect to $x$, when the applied load $q=0$, as

$$
\left[\begin{array}{lllll}
S_{11}^{(1)} & S_{12}^{(1)} & S_{13}^{(1)} & S_{14}^{(1)} & S_{15}^{(1)} \\
S_{21}^{(1)} & S_{22}^{(1)} & S_{23}^{(1)} & S_{24}^{(1)} & S_{25}^{(1)} \\
S_{31}^{(1)} & S_{32}^{(1)} & S_{33}^{(1)} & S_{34}^{(1)} & S_{35}^{(1)} \\
S_{41}^{(1)} & S_{42}^{(1)} & S_{43}^{(1)} & S_{44}^{(1)} & S_{45}^{(1)} \\
S_{51}^{(1)} & S_{52}^{(1)} & S_{53}^{(1)} & S_{54}^{(1)} & S_{55}^{(1)}
\end{array}\right]\left\{\begin{array}{c}
U_{n}^{(1)} \\
V_{n}^{(1)} \\
W_{n}^{(1)} \\
X_{n}^{(1)} \\
Y_{n}^{(1)}
\end{array}\right\}=\left\{\begin{array}{l}
0 \\
0 \\
0 \\
0 \\
0
\end{array}\right\},
$$

where elements in the matrix are presented in [6]. We have the following equation

$$
c_{0}+c_{1} \lambda_{n}^{2}+c_{2} \lambda_{n}^{4}+c_{3} \lambda_{n}^{6}+c_{4} \lambda_{n}^{8}+c_{5} \lambda_{n}^{10}=0,
$$

where coefficients $c_{i}, i=0,1,2,3,4,5$, can be obtained by expansion of determinant of matrix in (16) in the order of $\lambda_{n}$ by the mathematical tool such as Maple. For example, one of the constants in (17)

$$
c_{5}=\kappa A_{55}\left(A_{11} A_{66} D_{11} D_{66}+B_{11}^{2} B_{66}^{2}-B_{11}^{2} A_{66} D_{66}-A_{11} B_{66}^{2} D_{11}\right) .
$$

Other coefficients are too lengthy to be listed here. However, we can determine these coefficients easily by selecting arbitrary six values of $\bar{\lambda}_{k}, k=0,1,2, . .5$. Substituting the value of $\lambda$ into (17) gives

$$
c_{0}+c_{1} \bar{\lambda}_{k}^{2}+c_{2} \bar{\lambda}_{k}^{4}+c_{3} \bar{\lambda}_{k}^{6}+c_{4} \bar{\lambda}_{k}^{8}+c_{5} \bar{\lambda}_{k}^{10}=\left|S\left(\bar{\lambda}_{k}\right)\right|, \quad k=0,1,2, \ldots 5 .
$$

Then, six coefficients $c_{k}$ can be obtained. Substituting coefficients $c_{k}$ into (17), one can obtain ten roots $\lambda_{n i}\left(\beta_{n}\right), i=1,2, \ldots, 10$. For each root, $\lambda_{n i}$, let $A_{5 i}^{(1, n)}=1$ in (17) and we have four linear equations to determine rest constants $A_{j i}^{(1, n)}, j=1,2,3,4$, by

$$
\left[\begin{array}{llll}
S_{11}^{(1)} & S_{12}^{(1)} & S_{13}^{(1)} & S_{14}^{(1)} \\
S_{21}^{(1)} & S_{22}^{(1)} & S_{23}^{(1)} & S_{24}^{(1)} \\
S_{31}^{(1)} & S_{32}^{(1)} & S_{33}^{(1)} & S_{34}^{(1)} \\
S_{41}^{(1)} & S_{42}^{(1)} & S_{43}^{(1)} & S_{44}^{(1)}
\end{array}\right]\left\{\begin{array}{l}
A_{1 i}^{(1, n)} \\
A_{2 i}^{(1, n)} \\
A_{3 i}^{(1, n)} \\
A_{4 i}^{(1, n)}
\end{array}\right\}=-\left\{\begin{array}{l}
S_{15}^{(1)} \\
S_{25}^{(1)} \\
S_{35}^{(1)} \\
S_{45}^{(1)}
\end{array}\right\}, \quad \text { if } \operatorname{det}\left|\begin{array}{llll}
S_{11}^{(1)} & S_{12}^{(1)} & S_{13}^{(1)} & S_{14}^{(1)} \\
S_{21}^{(1)} & S_{22}^{(1)} & S_{23}^{(1)} & S_{24}^{(1)} \\
S_{31}^{(1)} & S_{32}^{(1)} & S_{33}^{(1)} & S_{34}^{(1)} \\
S_{41}^{(1)} & S_{42}^{(1)} & S_{43}^{(1)} & S_{44}^{(1)}
\end{array}\right| \neq 0 .
$$

Introducing a unit vector of coefficient in complex value as 


$$
e_{j, i}^{(1, n)}=\frac{A_{j i}^{(1, n)}}{A_{i}^{(1, n)}}, \quad A_{i}^{(1, n)}=\sqrt{\sum_{j=1}^{5}\left|A_{j i}^{(1, n)}\right|^{2}},
$$

then the general solution can be obtained by

$$
\left\{\begin{array}{c}
U_{n}^{(1)} \\
V_{n}^{(1)} \\
W_{n}^{(1)} \\
X_{n}^{(1)} \\
Y_{n}^{(1)}
\end{array}\right\}=\left[\begin{array}{cccccc}
e_{1,1}^{(1, n)} & e_{1,2}^{(1, n)} & \ldots & \ldots & \ldots & e_{1,10}^{(1, n)} \\
e_{2,1}^{(1, n)} & e_{2,2}^{(1, n)} & \ldots & \ldots & \ldots & e_{2,10}^{(1, n)} \\
e_{3,1}^{(1, n)} & e_{3,2}^{(1, n)} & \ldots & \ldots & \ldots & e_{3,10}^{(1, n)} \\
e_{4,1}^{(1, n)} & e_{4,2}^{(1, n)} & \ldots & \ldots & \ldots & e_{4, n)}^{(1, n)} \\
e_{5,1}^{(1, n)} & e_{5,2}^{(1, n)} & \ldots & \ldots & \ldots & e_{5,10}^{(1, n)}
\end{array}\right]\left\{\begin{array}{c}
B_{1}^{(n)} e^{\lambda_{n 1} x} \\
B_{2}^{(n)} e^{\lambda_{n 2} x} \\
\ldots \\
\ldots \\
B_{10}^{(n)} e^{\lambda_{n 10} x}
\end{array}\right\},
$$

where coefficients $B_{i}^{(n)}\left(\beta_{n}, s\right), i=1,2, \ldots, 10$, are unknowns in complex value. In the same way, the boundary conditions with other two opposite simply supported edges, defined as Case 2, can be described

$$
\begin{gathered}
v(0, y)=N_{1}(0, y)=w(0, y)=\varphi_{2}(0, y)=M_{1}(0, y)=0, \\
v(a, y)=N_{1}(a, y)=w(a, y)=\varphi_{2}(a, y)=M_{1}(a, y)=0 .
\end{gathered}
$$

Then the exact form of the spatial variations of displacement and rotation can be written as

$$
\begin{gathered}
u^{(2)}(x, y)=\sum_{m=1}^{\infty} U_{m}^{(2)}(y) \cos \alpha_{m} x, \\
v^{(2)}(x, y)=\sum_{m=1}^{\infty} V_{m}^{(2)}(y) \sin \alpha_{m} x, \\
w^{(2)}(x, y)=\sum_{m=1}^{\infty} W_{m}^{(2)}(y) \sin \alpha_{m} x, \\
\varphi_{1}^{(2)}(x, y)=\sum_{m=1}^{\infty} X_{m}^{(2)}(y) \cos \alpha_{m} x, \\
\varphi_{2}^{(2)}(x, y)=\sum_{m=1}^{\infty} Y_{m}^{(2)}(y) \sin \alpha_{m} x,
\end{gathered}
$$

where $\alpha_{m}=m \pi / a$. Assuming that the general solutions have the following form as

$$
\left\{\begin{array}{c}
U_{m}^{(2)} \\
V_{m}^{(2)} \\
W_{m}^{(2)} \\
X_{m}^{(2)} \\
Y_{m}^{(2)}
\end{array}\right\}=\left\{\begin{array}{l}
A_{1}^{(2, m)} \\
A_{2}^{(2, m)} \\
A_{3}^{(2, m)} \\
A_{4}^{(2, m)} \\
A_{5}^{(2, m)}
\end{array}\right\} e^{\gamma_{m}\left(\alpha_{m}\right) y}
$$

where $A_{i}^{(2, m)}$ are unknown coefficients. Same as Case 1, we have the following equation

$$
d_{0}+d_{1} \gamma_{m}^{2}+d_{2} \gamma_{m}^{4}+d_{3} \gamma_{m}^{6}+d_{4} \gamma_{m}^{8}+d_{5} \gamma_{m}^{10}=0
$$


where coefficients $d_{i}, i=0,1,2,3,4,5$, can be obtained by expansion of determinant of matrix in (16) in the order of $\gamma_{m}\left(\alpha_{m}\right)$. The general solution can be obtained by

$$
\left\{\begin{array}{l}
U_{m}^{(2)} \\
V_{m}^{(2)} \\
W_{m}^{(2)} \\
X_{m}^{(2)} \\
Y_{m}^{(2)}
\end{array}\right\}=\left[\begin{array}{llllll}
e_{1,1}^{(2, m)} & e_{1,2}^{(2, m)} & \ldots & \ldots & \ldots & e_{1,10}^{(2, m)} \\
e_{2,1}^{(2, m)} & e_{2,2}^{(2, m)} & \ldots & \ldots & \ldots & e_{2,10}^{(2, m)} \\
e_{3,1}^{(2, m)} & e_{3,2}^{(2, m)} & \ldots & \ldots & \ldots & e_{3,10}^{(2, m)} \\
e_{4,1}^{(2, m)} & e_{4,2}^{(2, m)} & \ldots & \ldots & \ldots & e_{4,10}^{(2, m)} \\
e_{5,1}^{(2, m)} & e_{5,2}^{(2, m)} & \ldots & \ldots & \ldots & e_{5,10}^{(2, m)}
\end{array}\right]\left\{\begin{array}{c}
C_{1}^{(m)} e^{\gamma_{m 1} x} \\
C_{2}^{(m)} e^{\gamma_{m 2} x} \\
\ldots \\
\ldots \\
C_{10}^{(m)} e^{\gamma_{m 10} x}
\end{array}\right\},
$$

where coefficients $C_{i}^{(m)}\left(\alpha_{m}, s\right), i=1,2, \ldots, 10$, are unknowns in complex value.

\subsection{Particular solution}

For general distribution of transverse load, such as a concentrated force, we can use the double Fourier transform method and obtain

$$
q(x, y)=\sum_{n=1}^{\infty} \sum_{m=1}^{\infty} q_{m n} \sin \alpha_{m} x \sin \beta_{n} y, \quad q_{m n}=\frac{4}{a b} \int_{0}^{a} \int_{0}^{b} q(x, y) \sin \alpha_{m} x \sin \beta_{n} y d x d y .
$$

Then the particular solution can be written as

$$
\begin{aligned}
& u^{*}(x, y, s)=\sum_{m=1}^{\infty} \sum_{n=1}^{\infty} U_{m n}^{*} \cos \alpha_{m} x \sin \beta_{n} y, \\
& v^{*}(x, y, s)=\sum_{m=1}^{\infty} \sum_{n=1}^{\infty} V_{m n}^{*} \sin \alpha_{m} x \cos \beta_{n} y, \\
& w^{*}(x, y, s)=\sum_{m=1}^{\infty} \sum_{n=1}^{\infty} W_{m n}^{*} \sin \alpha_{m} x \sin \beta_{n} y, \\
& \varphi_{1}^{*}(x, y, s)=\sum_{m=1}^{\infty} \sum_{n=1}^{\infty} X_{m n}^{*} \cos \alpha_{m} x \sin \beta_{n} y, \\
& \varphi_{2}^{*}(x, y, s)=\sum_{m=1}^{\infty} \sum_{n=1}^{\infty} Y_{m n}^{*} \sin \alpha_{m} x \cos \beta_{n} y .
\end{aligned}
$$

Five coefficients $\left\{U_{m n}^{*}, V_{m n}^{*}, W_{m n}^{*}, X_{m n}^{*}, Y_{m n}^{*}\right\}$ can be determined directly.

\subsection{Analytical solutions with four clamped edges}

According to the principal of superposition, the exact form of the spatial variation of displacement and rotation should be in the following form

$$
\begin{aligned}
& u(x, y)=\sum_{n=1}^{\infty} U_{n}^{(1)}(x) \sin \beta_{n} y+\sum_{m=1}^{\infty} U_{m}^{(2)}(y) \cos \alpha_{m} x+\sum_{m=1}^{\infty} \sum_{n=1}^{\infty} U_{m n}^{*} \cos \alpha_{m} x \sin \beta_{n} y, \\
& v(x, y)=\sum_{n=1}^{\infty} V_{n}^{(1)}(x) \cos \beta_{n} y+\sum_{m=1}^{\infty} V_{m}^{(2)}(y) \sin \alpha_{m} x+\sum_{m=1}^{\infty} \sum_{n=1}^{\infty} V_{m n}^{*} \sin \alpha_{m} x \cos \beta_{n} y,
\end{aligned}
$$




$$
\begin{aligned}
& w(x, y)=\sum_{n=1}^{\infty} W_{n}^{(1)}(x) \sin \beta_{n} y+\sum_{m=1}^{\infty} W_{m}^{(2)}(y) \sin \alpha_{m} x+\sum_{m=1}^{\infty} \sum_{n=1}^{\infty} W_{m n}^{*} \sin \alpha_{m} x \sin \beta_{n} y, \\
& \varphi_{1}(x, y)=\sum_{n=1}^{\infty} X_{n}^{(1)}(x) \sin \beta_{n} y+\sum_{m=1}^{\infty} X_{m}^{(2)}(y) \cos \alpha_{m} x+\sum_{m=1}^{\infty} \sum_{n=1}^{\infty} X_{m n}^{*} \cos \alpha_{m} x \sin \beta_{n} y, \\
& \varphi_{2}(x, y)=\sum_{n=1}^{\infty} Y_{n}^{(1)}(x) \cos \beta_{n} y+\sum_{m=1}^{\infty} Y_{m}^{(2)}(y) \sin \alpha_{m} x+\sum_{m=1}^{\infty} \sum_{n=1}^{\infty} Y_{m n}^{*} \sin \alpha_{m} x \cos \beta_{n} y,
\end{aligned}
$$

where coefficients $B_{i}\left(\beta_{n}\right), C_{i}\left(\alpha_{m}\right), i=1,2, \ldots, 10 ; m=1,2, \ldots, M$ and $n=1,2, \ldots, N$ are unknowns and $M$ and $N$ are the numbers of truncation term of Fourier series. Clamped edge boundary conditions indicate

$$
u=v=w=\varphi_{1}=\varphi_{2}=0,
$$

at all boundaries. Therefore, from (30) and (31), one obtains following linear equations

$$
\begin{cases}\sum_{i=1}^{10} e_{1, i}^{(1, n)} B_{i}^{(n)}+\sum_{m=1}^{M} \sum_{i=1}^{10} a_{m n i} e_{1, i}^{(2, m)} C_{i}^{(m)}+\sum_{n=1}^{L} U_{m n}^{*}=0 & \\ \sum_{i=1}^{\infty} e_{2, i}^{(1, n)} B_{i}^{(n)}=0 & n=1,2, \ldots, N \\ \sum_{i=1}^{10} e_{3, i}^{(1, n)} B_{i}^{(n)}=0 & \\ \sum_{i=1}^{10} e_{4, i}^{(1, n)} B_{i}^{(n)}+\sum_{m=1}^{M} \sum_{i=1}^{10} a_{m n i} e_{4, i}^{(2, m)} C_{i}^{(m)}+\sum_{m=1}^{L} X_{m n}^{*}=0 & \\ \sum_{i=1}^{10} e_{5, i}^{(1, n)} B_{i}^{(n)}=0 & \end{cases}
$$

for $x=0$, where $L$ is a large number (50 in all numerical examples) and

$$
a_{m n i}=\frac{2}{b} \frac{\beta_{n}}{\gamma_{m i}^{2}+\beta_{n}^{2}}\left[1-(-1)^{n} e^{\gamma_{m i} b}\right]
$$

For the second edge, one has

$$
\begin{cases}\sum_{i=1}^{\infty} e_{1, i}^{(2, m)} C_{i}^{(m)}=0 & \\ \sum_{n=1}^{N} \sum_{i=1}^{10} b_{m n i} e_{1, i}^{(1, n)} B_{i}^{(n)}+\sum_{i=1}^{10} e_{1, i}^{(2, m)} C_{i}^{(m)}+\sum_{n=1}^{L} V_{m n}^{*}=0 & m=1,2, \ldots, M \\ \sum_{i=1}^{10} e_{3, i}^{(2, m)} C_{i}^{(m)}=0 & \\ \sum_{i=1}^{10} e_{4, i}^{(2, m)} C_{i}^{(m)}=0 & \\ \sum_{n=1}^{N} \sum_{i=1}^{10} b_{m n i} e_{5, i}^{(1, n)} B_{i}^{(n)}+\sum_{i=1}^{10} e_{5, i}^{(2, m)} C_{i}^{(m)}+\sum_{n=1}^{L} Y_{m n}^{*}=0\end{cases}
$$


on $y=0$, where

$$
b_{m n i}=\frac{2}{a} \frac{\alpha_{m}}{\lambda_{n i}^{2}+\alpha_{m}^{2}}\left[1-(-1)^{m} e^{\lambda_{n i} a}\right] .
$$

For the third edge, we have

$$
\begin{cases}\sum_{i=1}^{10} e_{1, i}^{(1, n)} e^{\lambda_{m i} a} B_{i}^{(n)}+\sum_{m=1}^{M} \sum_{i=1}^{10}(-1)^{m} a_{m n i} e_{1, i}^{(2, m)} C_{i}^{(m)}+\sum_{m=1}^{L}(-1)^{m} U_{m n}^{*}=0 & \\ \sum_{i=1}^{\infty} e_{2, i}^{(1, n)} e^{\lambda_{n i} a} B_{i}^{(n)}=0 & n=1,2, \ldots, N, \\ \sum_{i=1}^{10} e_{3, i}^{(1, n)} e^{\lambda_{n i} a} B_{i}^{(n)}=0 & \\ \sum_{i=1}^{10} e_{4, i}^{(1, n)} e^{\lambda_{n i} a} B_{i}^{(n)}+\sum_{m=1}^{M} \sum_{i=1}^{10}(-1)^{m} a_{m n i} e_{4, i}^{(2, m)} C_{i}^{(m)}+\sum_{m=1}^{L}(-1)^{m} X_{m n}^{*}=0 & \\ \sum_{i=1}^{10} e_{5, i}^{(1, n)} e^{\lambda_{m i} a} B_{i}^{(n)}=0 & \end{cases}
$$

on $x=a$ and for the last edge

$$
\begin{cases}\sum_{i=1}^{\infty} e_{1, i}^{(2, m)} e^{\gamma_{m i} b} C_{i}^{(m)}=0 & m=1,2, \ldots, M, \\ \sum_{n=1}^{N} \sum_{i=1}^{10}(-1)^{n} b_{m n i} e_{1, i}^{(1, n)} e^{\gamma_{m i} b} B_{i}^{(n)}+\sum_{i=1}^{10} e_{1, i}^{(2, m)} e^{\gamma_{i} b} C_{i}^{(m)}+\sum_{n=1}^{L}(-1)^{n} V_{m n}^{*}=0 & \\ \sum_{i=1}^{10} e_{3, i}^{(2, m)} e^{\gamma_{m i} b} C_{i}^{(m)}=0 & \\ \sum_{i=1}^{10} e_{4, i}^{(2, m)} e^{\gamma_{m i} b} C_{i}^{(m)}=0 & \\ \sum_{n=1}^{N} \sum_{i=1}^{10}(-1)^{n} b_{m n i} e_{5, i}^{(1, n)} B_{i}^{(n)}+\sum_{i=1}^{10} e_{5, i}^{(2, m)} e^{\gamma_{m i} b} C_{i}^{(m)}+\sum_{n=1}^{L}(-1)^{n} Y_{m n}^{*}=0 & \end{cases}
$$

on $y=b$. We should have $10 \times(N+M)$ linear algebraic equations to determine the same number unknown coefficients, i.e. $B_{i}^{(n)}$ and $C_{i}^{(m)}$. Therefore, the analytical solutions for the rectangular double covered shells with four clamped edges are obtained.

\section{EXACT SOLUTION FOR DISTRIBUTED AND CONENTRATED FORCES}

One cross-ply laminates is considered $\left[0^{\circ} / 90^{\circ}\right]$ in this example with the same constants of material property in the above example. Truncation number of the Fourier series $(M, N)$ is taken to be 10 . Spherical and cylindrical shells are investigated with different boundary constrains under static transverse load $q_{0}$. Non-dimensional transverse deflection $\bar{w}_{0}\left(=w_{0} E_{2} h^{3} \times 10^{3} / q_{0} a^{4}\right)$, normalised resultants of stresses $\bar{N}_{i}\left(=N_{i} / q_{0} a\right)$ and $\bar{M}_{i}\left(=M_{i} / q_{0} a^{2}\right)$ at the centre of the shell are presented in Tables 1 and 2 respectively for various values of radius-to-side $(R / a)$ ratios and two values of thickness-to-side $(h / a)$ ratios. In addition, spherical and cylindrical shells subjected to a concentrated load $P_{0}$ are considered. In this 
case, the non-dimensional transverse deflection $\bar{w}_{0}\left(=w_{0} E_{2} h^{3} \times 10^{3} / P_{0} a^{2}\right)$, normalised resultants of stresses $\bar{N}_{i}\left(=N_{i} a / P_{0}\right)$ and $\bar{M}_{i}\left(=M_{i} / P_{0}\right)$ at the centre of the shell are presented in Table 3 for various values of radius-to-side $(R / a)$ ratios and two values of thickness-toside $(h / a)$ ratios. As the in-plane force and moment at the point of concentrated force are singular, they divergent with the number of truncation of Fourier series $(M, N)$. However, the resultants of stresses are regular and convergent in the domain of shell elsewhere.

\section{CONCLUSIONS}

In this paper, the exact solutions for the double curved laminated and functionally graded rectangular shells with four clamped were derived subjected to static distributed and concentrated transverse loads. The governing equations for the laminated and functionally graded shells with respect to the middle surface were presented with the moderate thick plate theory. The fundamental solution can be applied to the boundary element and the method of fundamental solution. In addition, these solutions can be used to assess the degree of accuracy for any numerical methods.

Table 1: Normalised deflection, in-plane force and moment for a laminated spherical shell $\left[0^{\circ} / 90^{\circ}\right]\left(R_{1}=R_{2}=R\right)$.

\begin{tabular}{cccccc|ccccc}
\hline \multirow{2}{*}{$R / a$} & \multicolumn{6}{c|}{$h / a=0.01$} & \multicolumn{5}{c}{$h / a=0.1$} \\
\cline { 2 - 10 } & $\bar{w}_{0}$ & $\bar{N}_{1}$ & $\bar{M}_{1}$ & $\bar{N}_{2}$ & $\bar{M}_{2}$ & $\bar{w}_{0}$ & $\bar{N}_{1}$ & $\bar{M}_{1}$ & $\bar{N}_{2}$ & $\bar{M}_{2}$ \\
\hline 1 & 0.0044 & 0.5006 & 0.0011 & 0.4990 & -0.0011 & 0.5456 & 0.5072 & 0.0126 & 0.5045 & -0.0102 \\
2 & 0.0187 & 1.0024 & 0.0023 & 0.9965 & -0.0023 & 1.9548 & 0.8283 & 0.0247 & 0.8201 & -0.0124 \\
3 & 0.0463 & 1.5320 & 0.0035 & 1.5223 & -0.0035 & 3.2671 & 0.9047 & 0.0313 & 0.8890 & -0.0092 \\
4 & 0.0908 & 2.0939 & 0.0048 & 2.0832 & -0.0046 & 4.2024 & 0.8684 & 0.0339 & 0.8469 & -0.0048 \\
5 & 0.1528 & 2.6645 & 0.0063 & 2.6536 & -0.0057 & 4.8277 & 0.7977 & 0.0346 & 0.7721 & -0.0008 \\
10 & 0.6511 & 5.0750 & 0.0144 & 5.0392 & -0.0085 & 5.9965 & 0.5013 & 0.0323 & 0.4681 & 0.0104 \\
$\infty$ & 3.9782 & 0.1817 & 0.0239 & -0.1817 & 0.0239 & 6.5150 & 0.0183 & 0.0233 & -0.0183 & 0.0233 \\
\hline
\end{tabular}

Table 2: Normalised deflection, in-plane force and moment for a laminated cylindrical shell $\left[0^{\circ} / 90^{\circ}\right]\left(R_{1}=\infty, R_{2}=R\right)$.

\begin{tabular}{cccccc|ccccc}
\hline & \multicolumn{6}{c|}{$h / a=0.01$} & \multicolumn{5}{c}{$h / a=0.1$} \\
\cline { 2 - 11 }$R / a$ & $\bar{w}_{0}$ & $\bar{N}_{1}$ & $\bar{M}_{1}$ & $\bar{N}_{2}$ & $\bar{M}_{2}$ & $\bar{w}_{0}$ & $\bar{N}_{1}$ & $\bar{M}_{1}$ & $\bar{N}_{2}$ & $\bar{M}_{2}$ \\
\hline 1 & 0.0135 & 0.0269 & 0.0000 & 1.0000 & -0.0022 & 1.1762 & 0.0246 & 0.0029 & 0.9075 & -0.0167 \\
2 & 0.0572 & 0.0555 & 0.0001 & 1.9880 & -0.0042 & 3.1310 & 0.0360 & 0.0100 & 1.2210 & -0.0170 \\
3 & 0.1306 & 0.0854 & 0.0001 & 3.0104 & -0.0061 & 4.4235 & 0.0377 & 0.0150 & 1.1500 & -0.0108 \\
4 & 0.2338 & 0.1163 & 0.0004 & 4.0588 & -0.0079 & 5.1545 & 0.0364 & 0.0179 & 1.0026 & -0.0049 \\
5 & 0.3630 & 0.1468 & 0.0009 & 5.0709 & -0.0094 & 5.5775 & 0.0346 & 0.0196 & 0.8652 & -0.0003 \\
10 & 1.1995 & 0.2653 & 0.0057 & 8.4974 & -0.0124 & 6.2562 & 0.0281 & 0.0223 & 0.4767 & 0.0110 \\
$\infty$ & 3.9782 & 0.1817 & 0.0239 & -0.1817 & 0.0239 & 6.5150 & 0.0183 & 0.0233 & -0.0183 & 0.0233 \\
\hline
\end{tabular}


Table 3: Normalised deflection $\bar{w}_{0}$ for a laminated spherical shell $\left[0^{\circ} / 90^{\circ}\right]$.

\begin{tabular}{cccccc|ccccc}
\hline & \multicolumn{5}{c|}{ Spherical shell $\left(R_{1}=R_{2}=R\right)$} & \multicolumn{5}{c}{ Cylindrical shell $\left(R_{1}=\infty, R_{2}=R\right)$} \\
\cline { 2 - 11 }$R / a$ & $h / a=0.01$ & 0.05 & 0.1 & 0.15 & 0.2 & 0.01 & 0.05 & 0.1 & 0.15 & 0.2 \\
\hline 1 & 0.0044 & 0.5006 & 0.0011 & 0.4990 & -0.0011 & 0.5456 & 0.5072 & 0.0126 & 0.5045 & -0.0102 \\
2 & 0.0187 & 1.0024 & 0.0023 & 0.9965 & -0.0023 & 1.9548 & 0.8283 & 0.0247 & 0.8201 & -0.0124 \\
3 & 0.0463 & 1.5320 & 0.0035 & 1.5223 & -0.0035 & 3.2671 & 0.9047 & 0.0313 & 0.8890 & -0.0092 \\
4 & 0.0908 & 2.0939 & 0.0048 & 2.0832 & -0.0046 & 4.2024 & 0.8684 & 0.0339 & 0.8469 & -0.0048 \\
5 & 0.1528 & 2.6645 & 0.0063 & 2.6536 & -0.0057 & 4.8277 & 0.7977 & 0.0346 & 0.7721 & -0.0008 \\
10 & 0.6511 & 5.0750 & 0.0144 & 5.0392 & -0.0085 & 5.9965 & 0.5013 & 0.0323 & 0.4681 & 0.0104 \\
$\infty$ & 3.9782 & 0.1817 & 0.0239 & 0.1817 & 0.0239 & 6.5150 & 0.0183 & 0.0233 & -0.0183 & 0.0233 \\
\hline
\end{tabular}

\section{REFERENCES}

[1] Miyamoto, Y. et al., Functionally Graded Materials: Design, Processing and Applications, Kluwer: Boston, 1992.

[2] Reddy, J.N., Exact solutions of moderately thick laminated shells. Journal of Engineering Mechanics, 110(5), pp. 749-809, 1984. DOI: 10.1061/(asce)0733-9399 (1984)110:5(794).

[3] Reddy, J.N. \& Liu, C.F., A higher-order shear deformation theory of laminated elastic shells. International Journal of Engineering Science, 23(3), pp. 319-30, 1985.

DOI: 10.1016/0020-7225(85)90051-5.

[4] Sladek, J., Sladek, V., Zhang, Ch., Krivacek, J. \& Wen, PH., Analysis of orthotropic thick plates by meshless local Petrov-Galerkin (MLPG) method. International Journal for Numerical Methods in Engineering, 67(13), pp. 1830-1850, 2006.

DOI: $10.1002 / n m e .1683$.

[5] Reddy, J.N., Bending of laminated anisotropic shells by a shear deformable finite element. Fibre Science and Technology, 17(1), pp. 9-24, 1982. DOI: 10.1016/00150568(82)90058-6.

[6] Wen, P.H. \& Aliabadi, M.H., On the theory of functionally graded moderately thick composite shells. Journal of Multiscale Modelling, 5(3), p. 1350012, 2013.

DOI: $10.1142 / \mathrm{s} 1756973713500121$. 